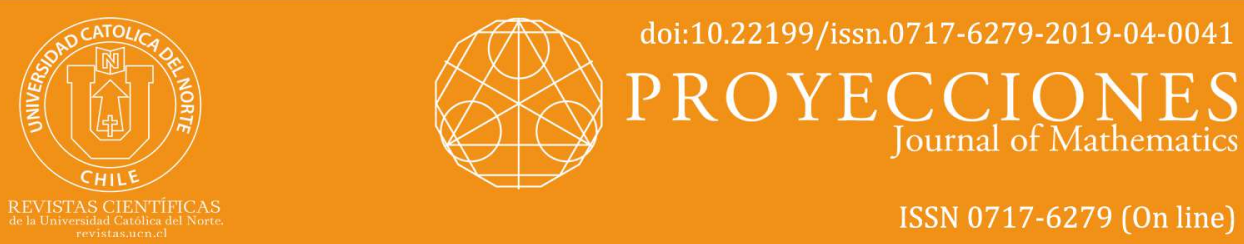

\title{
A relation of Banach limit and difference matrix to generate some Orlicz sequence spaces
}

Kuldip Raj* (D) orcid.org/0000-0002-2611-3391

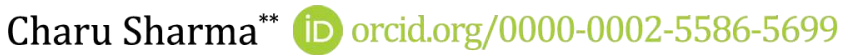

*Shri Mata Vaishno Devi University, School of Mathematics. Katra, JK, India.

kuldipraj68@gmail.com

* Shri Mata Vaishno Devi University, School of Mathematics. Katra, JK, India

诖 charu145.cs@gmail.com

Received: March 2018 | Accepted: July 2019

\section{Abstract:}

In the present paper we study the applications of generalized difference matrices and Orlicz function to generate some spaces of almost convergent sequences. We make an effort to study some algebraic and topological properties of these sequence spaces. Some inclusión relations between these spaces are establish. Furthermore, we study matrix transformations and compute $\beta-, \gamma-$ duals of these spaces.

Keywords: Paranorm space; Orlicz function; $\beta$-and $\gamma$-duals; Matrix transformations.

$\operatorname{MSC}(2010): 40 \mathrm{C05}, 40 \mathrm{J05}, 46 \mathrm{~A} 45$.

\section{Cite this article as (IEEE citation style):}

K. Raj and C. Sharma, "A relation of Banach limit and difference matrix to generate some Orlicz sequence spaces", Proyecciones (Antofagasta, On line), vol. 38, no. 4, pp. 637-652, Oct. 2019, doi: 10.22199/issn.0717-6279-2019-04-0041. [Accessed dd-mm-yyyy].

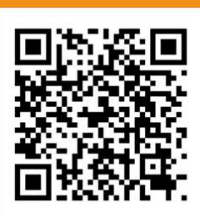

Article copyright: (C) 2019 Kudilp Raj and Charu Sharma. This is an open access article distributed under the terms of the Creative Commons Licence, which permits unrestricted use and distribution provided the original author and source are credited. 


\section{Introduction and Preliminaries}

The notion of almost convergence was introduced by Lorentz [7]. Matrix domains of the generalized difference matrix $B(r, s)$ and triple band matrix $B(r, s, t)$ in sets of almost null and almost convergent sequences have been investigated by Başar and Kirişçi [3] and Sönmez [18], respectively. Let $w$ be the vector space of all real sequences. We shall write $c, c_{0}$ and $l_{\infty}$ for the spaces of all convergent, null and bounded sequences. Moreover, we write $b s$ and $c s$ for the spaces of all bounded and convergent series, respectively. Let $X$ and $Y$ be two sequence spaces and $A=\left(a_{n k}\right)$ be an infinite matrix of real or complex entries, where $n, k \in \mathbf{N}$. Then we say that $A$ defines a matrix mapping from $X$ into $Y$ if for every sequence $x=\left(x_{k}\right) \in X$, the sequence $A x=\left\{A_{n}(x)\right\}$ is in $Y$, where

$$
A_{n}(x)=\sum_{k} a_{n k} x_{k} \quad(n \in \mathbf{N}) .
$$

By $(X, Y)$ we denote the class of all matrices $A$ such that $A: X \rightarrow Y$. Thus $A \in(X, Y)$ if and only if the series on the right-hand side of 1.1 converges for each $n \in \mathbf{N}$ and every $x \in X$ and we have $A x \in Y$ for all $x \in X$.

The matrix domain $X_{A}$ of an infinite matrix $A$ in a sequence space $X$ is defined by

$$
X_{A}=\left\{x=\left(x_{k}\right) \in w: A x \in X\right\}
$$

which is a sequence space.

A $B$-space is a complete normed space. A topological sequence space in which all coordinate functionals $\pi_{k}, \pi_{k}(x)=x_{k}$, are continuous is called a $K$-space. A $B K$-space is defined as a $K$-space which is also a $B$-space, that is, a $B K$-space is a Banach space with continuous coordinates. For example, the space $l_{p}(1 \leq p<\infty)$ is a $B K$-space with $\|x\|_{p}=\left(\sum_{k=0}^{\infty}\left|x_{k}\right|^{p}\right)^{\frac{1}{p}}$ and $c_{0}, c$ and $l_{\infty}$ are $B K$-spaces with $\|x\|_{\infty}=\sup _{k}\left|x_{k}\right|$. A sequence $\left(b_{n}\right)$ in a normed space $X$ is called a Schauder basis for $X$ if for every $x \in X$ there is a unique sequence $\left(\alpha_{n}\right)$ of scalars such that $x=\sum_{n} \alpha_{n} b_{n}$, i.e., $\lim _{m}\left\|x-\sum_{n=0}^{m} \alpha_{n} b_{n}\right\|=0$. 
The Cesàro matrix $C=\left(c_{n k}\right)$ of order one is a triangle matrix defined by

$$
c_{n k}= \begin{cases}\frac{1}{n+1}, & 0 \leq k \leq n \\ 0, & k>n\end{cases}
$$

for all $n, k \in \mathbf{N}$.

One of the best known regular matrix is the Riesz matrix $R=\left(r_{n k}\right)$, which is a triangle matrix and is defined by

$$
r_{n k}= \begin{cases}\frac{r_{k}}{R_{n}}, & 0 \leq k \leq n, \\ 0, & k>n,\end{cases}
$$

for all $n, k \in \mathbf{N}$, where $\left(r_{k}\right)$ is a real sequence with $r_{0}>0, r_{k} \geq 0$ and $R_{n}=\sum_{k=0}^{n} r_{k}$. The Riesz matrix $R$ is regular if and only if $R_{n} \rightarrow \infty$ as $n \rightarrow \infty$ [13]. The matrix domain $X_{A}$ of a sequence space $X$ has a basis if and only if $X$ has a basis and $A=\left(a_{n k}\right)$ is a triangle matrix.

Let $r, s$ be non-zero real numbers and define the generalized difference matrix $B(r, s)=\left(b_{n k}(r, s)\right)$ for all $k, n \in \mathbf{N}$ as follows:

$$
b_{n k}(r, s)= \begin{cases}r, & k=n \\ s, & k=n-1 \\ 0, & 1 \leq k \leq n-1 \text { or } k>n\end{cases}
$$

It is easy to calculate that the inverse $B^{-1}(r, s)=\left(\hat{b}_{n k}(r, s)\right)$ of the generalized difference matrix is given by

$$
\hat{b}_{n k}(r, s)= \begin{cases}\frac{1}{r}\left(-\frac{s}{r}\right)^{n-k}, & 1 \leq k \leq n, \\ 0, & k>n\end{cases}
$$

for all $k, n \in \mathbf{N}$.

We now focus on sets of almost convergent sequences. A continuous linear functional $\phi$ on $l_{\infty}$ is called a Banach limit if

(i) $\phi(x) \geq 0$ for $x=\left(x_{k}\right), x_{k} \geq 0$ for every $\mathrm{k}$,

(ii) $\phi\left(x_{\sigma(k)}\right)=\phi\left(x_{k}\right)$, where $\sigma$ is shift operator which is defined on $w$ by $\sigma(k)=k+1$ and 
(iii) $\phi(e)=1$, where $e=(1,1,1, \ldots)$.

A sequence $x=\left(x_{k}\right) \in l_{\infty}$ is said to be almost convergent to the generalized limit $a$ if all Banach limits of $x$ are $a$ (see [7]) and denoted by $f-\lim x=a$. In other words, $f-\lim x_{k}=a$ uniformly in $n$ if and only if $\lim _{p \rightarrow \infty} \frac{\left(x_{n}+x_{n+1}+\ldots+x_{n+p-1}\right)}{p}=a$, uniformly in $n$. We denote the space of all almost convergent and almost null sequences by $f$ and $f_{0}$, respectively.

In [21] Zararsız and Şengönül defined the concepts of the spaces of $r f$ convergent and $r f$-null sequences and it is proved that the spaces $r f$ and $r f_{0}$ are Banach spaces with the norm

$$
\|x\|_{r f}=\|x\|_{r f_{0}}=\sup _{m}\left|\frac{1}{R_{m}} \sum_{k=0}^{m} r_{k} x_{k+n}\right| \text {, uniformly in } n \text {. }
$$

In addition to these spaces, Zararsiz [22] introduced two convergent sequences $\operatorname{brf}$ and $\operatorname{br} f_{0}$ as the sets of all sequences such that their $B(r, s)$ transforms are in the spaces $r f$ and $r f_{0}$, respectively.

Let us define the sequence $z=\left(z_{k}\right)$ as the $B(r, s)$-transform of a sequence $x=\left(x_{k}\right)$, that is,

$$
z_{k}=s x_{k-1}+r x_{k} \quad(k \in \mathbf{N}) .
$$

Corollary 1.1. [22] The space brf does not have a Schauder basis.

A set $\lambda \subset w$ is said to be convex if $x, y \in \lambda$ implies $C=\{z \in w: z=$ $t x+(1-t) y, 0 \leq t \leq 1\} \subset \lambda$.

An Orlicz function $M:[0, \infty) \rightarrow[0, \infty)$ is a continuous, non-decreasing and convex function such that $M(0)=0, M(x)>0$ for $x>0$ and $M(x) \longrightarrow \infty$ as $x \longrightarrow \infty$. Lindenstrauss and Tzafriri [6] used the idea of Orlicz function to define the following sequence space,

$$
\ell_{M}=\left\{x=\left(x_{k}\right) \in w: \sum_{k=1}^{\infty} M\left(\frac{\left|x_{k}\right|}{\rho}\right)<\infty, \text { for some } \rho>0\right\}
$$

is known as an Orlicz sequence space. The space $\ell_{M}$ is a Banach space with the norm

$$
\|x\|=\inf \left\{\rho>0: \sum_{k=1}^{\infty} M\left(\frac{\left|x_{k}\right|}{\rho}\right) \leq 1\right\} .
$$


A sequence $\mathcal{M}=\left(M_{k}\right)$ of Orlicz functions is said to be Musielak-Orlicz function (see [9], [12]). A Musielak-Orlicz function $\mathcal{M}=\left(M_{k}\right)$ is said to satisfy $\Delta_{2}$-condition if there exist constants $a, K>0$ and a sequence $c=\left(c_{k}\right)_{k=1}^{\infty} \in l_{+}^{1}$ (the positive cone of $\left.l^{1}\right)$ such that the inequality

$$
M_{k}(2 u) \leq K M_{k}(u)+c_{k}
$$

holds for all $k \in \mathbf{N}$ and $u \in R^{+}$, whenever $M_{k}(u) \leq a$. The reader can refer to the textbook Başar [2] containing the chapters entitled Normed and Paranormed Sequence Spaces and Matrix Domains in Sequence Spaces together with the paper Dutta and Başar [4] devoted to the generalization of Orlicz sequence spaces. For more details about sequence spaces see For more details about sequence spaces see ([10], [11], [14], [15], [16], [17], [20]) and references therein.

Definition 1.2. Let $X$ be a linear metric space. A function $p: X \rightarrow \mathbf{R}$ is called paranorm, if

$(P 1) p(x) \geq 0$ for all $x \in X$,

$(P 2) p(-x)=p(x)$ for all $x \in X$,

$(P 3) p(x+y) \leq p(x)+p(y)$ for all $x, y \in X$,

(P4) if $\left(\lambda_{n}\right)$ is a sequence of scalars with $\lambda_{n} \rightarrow \lambda$ as $n \rightarrow \infty$ and $\left(x_{n}\right)$ is a sequence of vectors with $p\left(x_{n}-x\right) \rightarrow 0$ as $n \rightarrow \infty$, then $p\left(\lambda_{n} x_{n}-\lambda x\right) \rightarrow$ 0 as $n \rightarrow \infty$.

A paranorm $p$ for which $p(x)=0$ implies $x=0$ is called total paranorm and the pair $(X, p)$ is called a total paranormed space. It is well known that the metric of any linear metric space is given by some total paranorm (see [19, Theorem 10.4.2, p. 183]).

Let $\mathcal{M}=\left(M_{k}\right)$ be a sequence of Orlicz functions, $p=\left(p_{k}\right)$ be any bounded sequence of positive real numbers and $u=\left(u_{k}\right)$ be a sequence of strictly positive real numbers. By making the use of $B(r, s)$-transform of sequences $x=\left(x_{k}\right)$, we define the following sequence spaces:

$$
\begin{gathered}
{[\operatorname{brf}, \mathcal{M}, u, p]=\left\{x=\left(x_{k}\right) \in w: \exists a \in \mathbf{C} \ni \lim _{m} \frac{1}{R_{m}} \sum_{k=0}^{m}\left[u_{k} M_{k}\left(\frac{r_{k}\left|s x_{k+n-1}+r x_{k+n}\right|}{\rho}\right)\right]^{p_{k}}=\right.} \\
a, \text { uniformily in } n \text { and some } \rho>0\}
\end{gathered}
$$


and

$$
\begin{gathered}
{\left[b r f_{0}, \mathcal{M}, u, p\right]=\left\{x=\left(x_{k}\right) \in w: \lim _{m} \frac{1}{R_{m}} \sum_{k=0}^{m}\left[u_{k} M_{k}\left(\frac{r_{k}\left|s x_{k+n-1}+r x_{k+n}\right|}{\rho}\right)\right]^{p_{k}}=0,\right.} \\
\text { uniformily in } n \text { and some } \rho>0\} .
\end{gathered}
$$

If $M_{k}(x)=x$ for all $k \in \mathbf{N}$ and $\rho=1$, then above sequence spaces reduces to $[b r f, u, p]$ and $\left[b r f_{0}, u, p\right]$, where

$$
\begin{gathered}
{[b r f, u, p]=\left\{x=\left(x_{k}\right) \in w: \exists a \in \mathbf{C} \ni \lim _{m} \frac{1}{R_{m}} \sum_{k=0}^{m}\left[u_{k}\left(r_{k}\left|s x_{k+n-1}+r x_{k+n}\right|\right)\right]^{p_{k}}=a,\right.} \\
\text { uniformily in } n\}
\end{gathered}
$$

and

$$
\begin{gathered}
{\left[b r f_{0}, u, p\right]=\left\{x=\left(x_{k}\right) \in w: \lim _{m} \frac{1}{R_{m}} \sum_{k=0}^{m}\left[u_{k}\left(r_{k}\left|s x_{k+n-1}+r x_{k+n}\right|\right)\right]^{p_{k}}=0,\right.} \\
\text { uniformily in } n\} .
\end{gathered}
$$

By taking $\left(p_{k}\right)=1$ and $\left(u_{k}\right)=1$, for all $k \in \mathbf{N}$, then we get the following sequence spaces:

$$
\begin{gathered}
{[b r f, \mathcal{M}]=\left\{x=\left(x_{k}\right) \in w: \exists a \in \mathbf{C} \ni \lim _{m} \frac{1}{R_{m}} \sum_{k=0}^{m}\left[M_{k}\left(\frac{r_{k}\left|s x_{k+n-1}+r x_{k+n}\right|}{\rho}\right)\right]=a,\right.} \\
\text { uniformily in } n \text { and some } \rho>0\}
\end{gathered}
$$


and

$$
\begin{gathered}
{\left[b r f_{0}, \mathcal{M}\right]=\left\{x=\left(x_{k}\right) \in w: \lim _{m} \frac{1}{R_{m}} \sum_{k=0}^{m}\left[M_{k}\left(\frac{r_{k}\left|s x_{k+n-1}+r x_{k+n}\right|}{\rho}\right)\right]=0,\right.} \\
\text { uniformily in } n \text { and some } \rho>0\} .
\end{gathered}
$$

With the notation of 1.1 , the sequence spaces $[b r f, \mathcal{M}, u, p]$ and $\left[b r f_{0}, \mathcal{M}, u, p\right]$ can be redefined as follows:

$\left[b r f_{0}, \mathcal{M}, u, p\right]=\left\{\left[r f_{0}, \mathcal{M}, u, p\right]\right\}_{B(r, s)}$ and $[b r f, \mathcal{M}, u, p]=\{[r f, \mathcal{M}, u, p]\}_{B(r, s)}$.

The following inequality will be use throughout the paper. If $0 \leq p_{k} \leq$ $\sup p_{k}=H, K=\max \left(1,2^{H-1}\right)$, then

$$
\left|a_{k}+b_{k}\right|^{p_{k}} \leq K\left\{\left|a_{k}\right|^{p_{k}}+\left|b_{k}\right|^{p_{k}}\right\}
$$

for all $k$ and $a_{k}, b_{k} \in \mathbf{C}$. Also $|a|^{p_{k}} \leq \max \left(1,|a|^{H}\right)$ for all $a \in \mathbf{C}$.

In this paper, we introduce the sequence spaces $[b r f, \mathcal{M}, u, p]$ and $\left[b r f_{0}, \mathcal{M}, u, p\right]$. We investigate some topological properties of these new sequence spaces and establish some inclusion relations between these spaces. Also we determine the $\alpha-, \beta-$ and $\gamma-$ duals of these spaces and construct the matrix transformation of these spaces.

\section{Main Results}

Theorem 2.1. Let $\mathcal{M}=\left(M_{k}\right)$ be a sequence of Orlicz functions, $p=\left(p_{k}\right)$ be a bounded sequence of positive real numbers and $u=\left(u_{k}\right)$ be a sequence of strictly positive real numbers. Then $[b r f, \mathcal{M}, u, p]$ and $\left[b r f_{0}, \mathcal{M}, u, p\right]$ are linear spaces over the complex field $\mathbf{C}$.

Proof. It is a routine verification so we omit the proof.

Theorem 2.2. Let $\mathcal{M}=\left(M_{k}\right)$ be a sequence of Orlicz functions and $p=\left(p_{k}\right)$ be a bounded sequence of positive real numbers and $u=\left(u_{k}\right)$ 
be a sequence of strictly positive real numbers. Then $[b r f, \mathcal{M}, u, p]$ and $\left[b r f_{0}, \mathcal{M}, u, p\right]$ are paranormed spaces with the paranorm defined by

$$
\begin{gathered}
g(x)=\inf \left\{(\rho)^{\frac{p_{k}}{M}}:\left(\frac{1}{R_{m}} \sum_{k=0}^{m}\left[u_{k} M_{k}\left(\frac{r_{k}\left|s x_{k+n-1}+r x_{k+n}\right|}{\rho}\right)\right]^{p_{k}}\right)^{\frac{1}{M}} \leq 1,\right. \\
\text { uniformly in } n>0, \quad \rho>0\},
\end{gathered}
$$

where $0 \leq p_{k} \leq \sup p_{k}=H, \quad M=\max (1, H)$.

Proof. For the proof verification see [15].

Theorem 2.3. Let $\mathcal{M}=\left(M_{k}\right)$ be a sequence of Orlicz functions, $u=\left(u_{k}\right)$ be a sequence of strictly positive real numbers. If $p=\left(p_{k}\right)$ and $q=\left(q_{k}\right)$ are bounded sequences of positive real numbers with $0 \leq p_{k} \leq q_{k}<\infty$ for each $k$, then $\left[b r f_{0}, \mathcal{M}, u, p\right] \subseteq[b r f, \mathcal{M}, u, q]$.

Proof. It is easy to prove.

Theorem 2.4. Let $\mathcal{M}=\left(M_{k}\right)$ be a sequence of Orlicz functions which satisfies the $\Delta_{2}$-condition and $\beta=\lim _{t \rightarrow \infty} \frac{M_{k}(t)}{t}>0$ for all $k \in \mathbf{N}$. Then $\left[b r f_{0}, \mathcal{M}, u, p\right] \subseteq\left[b r f_{0}, u, p\right]$.

Proof. It is easy to prove.

The following theorems can be proved in a similar way as in [15].

Theorem 2.5. If $\mathcal{M}^{\prime}=\left(M_{k}^{\prime}\right)$ and $\mathcal{M}^{\prime \prime}=\left(M_{k}^{\prime \prime}\right)$ are sequences of Orlicz functions satisfying the $\Delta_{2}$-condition, then

$$
\left[b r f_{0}, \mathcal{M}, u, p\right] \cap\left[b r f_{0}, \mathcal{M}^{\prime}, u, p\right] \subseteq\left[b r f_{0},\left(\mathcal{M}^{\prime}+\mathcal{M}^{\prime \prime}\right), u, p\right]
$$

Theorem 2.6. Let $\mathcal{M}=\left(M_{k}\right)$ and $\mathcal{M}^{\prime}=\left(M_{k}^{\prime}\right)$ be two sequences of Orlicz functions, then

$$
\left[b r f_{0}, \mathcal{M}^{\prime}, u, p\right] \subseteq\left[b r f_{0}, \mathcal{M} \circ \mathcal{M}^{\prime}, u, p\right]
$$

where $\mathcal{M} \circ \mathcal{M}^{\prime}$ is the composition of $\mathcal{M}$ and $\mathcal{M}^{\prime}$. 
Theorem 2.7. The spaces $[b r f, \mathcal{M}, u, p]$ and $\left[b r f_{0}, \mathcal{M}, u, p\right]$ are $B K$-spaces with the norm defined by

$$
\begin{gathered}
\|x\|_{b r f_{0}, \mathcal{M}, u, p}=\|x\|_{b r f, \mathcal{M}, u, p} \\
=\sup _{m}\left|\frac{1}{R_{m}} \sum_{k=0}^{m}\left[u_{k} M_{k}\left(\frac{r_{k}\left|s x_{k+n-1}+r x_{k+n}\right|}{\rho}\right)\right]^{p_{k}}\right| \text {, uniformly in } n .
\end{gathered}
$$

Proof. Since 1.4 holds, $b r f$ and $b r f_{0}$ are the BK-spaces with respect to their norms (see Theorem 3.3 in [22]) and the matrix $B(r, s)$ is normal, Theorem 4.3.12 of Wilansky [19] gives the fact that $[b r f, \mathcal{M}, u, p]$ and $\left[b r f_{0}, \mathcal{M}, u, p\right]$ are BK-spaces with the given norms. This completes the proof.

Theorem 2.8. The spaces $[b r f, \mathcal{M}, u, p]$ and $\left[b r f_{0}, \mathcal{M}, u, p\right]$ are linearly isomorphic to the spaces brf and br $f_{0}$, respectively.

Proof. We only consider the sequence spaces $[b r f, \mathcal{M}, u, p]$ and $b r f$ and other will follow similarly. To show the theorem, we must show the existence of linear bijection between the spaces $[b r f, \mathcal{M}, u, p]$ and $b r f$. For this, we consider the transformation $T$ defined with the notation 1.4, from $[\operatorname{brf}, \mathcal{M}, u, p]$ to $\operatorname{brf}$ by $x \rightarrow y=T x$. The linearity of $T$ is obvious. Further, it is trivial that $x=\theta=(0,0,0 \ldots)$ whenever $T x=\theta$ and hence $T$ is injective. Next, let $y=\left(y_{k}\right) \in \operatorname{brf}$ and defined the sequence $x=\left(x_{k}\right)$ by $\left(\left\{B^{-1}(r, s) y\right\}\right)_{k}$ for all $k \in \mathbf{N}$. Then, it is clear that

$\left\{B^{-1}(r, s) y\right\}_{k}=s x_{k-1}+r x_{k}=\sum_{j=0}^{k-1} \frac{s}{r}\left(-\frac{s}{r}\right)^{j} y_{k-j-1}+\sum_{j=0}^{k-1}\left(-\frac{s}{r}\right)^{j} y_{k-j}=y_{k}$

for all $k \in \mathbf{N}$ which shows that

$$
\begin{aligned}
\lim _{m} \frac{1}{R_{m}} \sum_{k=0}^{m}\left[u_{k} M_{k}\right. & \left.\left(\frac{r_{k}\left|s x_{k+n-1}+r x_{k+n}\right|}{\rho}\right)\right]^{p_{k}} \\
& =\lim _{m} \frac{1}{R_{m}} \sum_{k=0}^{m}\left[u_{k} M_{k}\left(\frac{r_{k}\left|y_{k+n}\right|}{\rho}\right)\right]^{p_{k}} \\
& =[b r f, \mathcal{M}, u, p]-\lim y_{k}, \text { uniformly in n. }
\end{aligned}
$$


Thus, $x=\left(x_{k}\right) \in[b r f, \mathcal{M}, u, p]$. Consequently, it is clear that $T$ is surjective. Because of the fact that is linear bijection, $[b r f, \mathcal{M}, u, p]$ and brf are linearly isomorphic. This completes the proof.

Theorem 2.9. The spaces $\left[b r f_{0}, \mathcal{M}, u, p\right]$ and $[b r f, \mathcal{M}, u, p]$ are convex spaces.

Proof. The proof is clear from the definition of convexity.

Corollary 2.10. The space $[b r f, \mathcal{M}, u, p]$ does not have a Schauder basis.

\section{3. $\beta$ and $\gamma$-Duals}

In this section, we determine the $\beta$ and $\gamma$-duals of the spaces $[b r f, \mathcal{M}, u, p]$ and $\left[b r f_{0}, \mathcal{M}, u, p\right]$. For the sequence spaces $X$ and $Y$, define the set $S(X, Y)$ by

$$
S(X, Y)=\left\{z=\left(z_{k}\right) \in w: x z=\left(x_{k} z_{k}\right) \in Y \text { for all } x=\left(x_{k}\right) \in X\right\} .
$$

With the notation of 3.1 the $\alpha$-, $\beta$ - and $\gamma$-duals of a sequence space $X$, which are, respectively, denoted by $X^{\alpha}, X^{\beta}$ and $X^{\gamma}$ are defined by $S\left(X, l_{1}\right)$, $S(X, c s)$ and $S(X, b s)$.

The following theorems are proved by using some lemmas of [21].

Theorem 3.1. The $\gamma$-dual of the space $[b r f, \mathcal{M}, u, p]$ is the set $d_{1}(r, s)$, where

$d_{1}(r, s)=\left\{a=\left(a_{k}\right) \in w: \sup _{n} \sum_{k=0}^{n}\left[u_{k} M_{k}\left(\frac{\left|\sum_{j=k}^{n} \frac{1}{r}\left(-\frac{s}{r}\right)^{j-k} a_{j}\right|}{\rho}\right)\right]^{p_{k}}<\infty\right\}$.

Proof. The proof of the theorem is clear, so we omit it.

Theorem 3.2. Let us write the sets $d_{2}(r, s), d_{3}(r, s)$ and $d_{4}(r, s)$ by

$$
\begin{aligned}
& d_{2}(r, s)=\left\{a=\left(a_{k}\right) \in w: \lim _{n} \sum_{k=0}^{n}\left[u_{k} M_{k}\left(\frac{\left|\sum_{j=k}^{n} \frac{1}{r}\left(-\frac{s}{r}\right)^{j-k} a_{j}\right|}{\rho}\right)\right]^{p_{k}} \text { exists }\right\}, \\
& d_{3}(r, s)=\left\{a=\left(a_{k}\right) \in w: \lim _{n} \sum_{k=0}^{n}\left[u_{k} M_{k}\left(\frac{\left|\Delta\left(\sum_{j=k}^{n} \frac{1}{r}\left(-\frac{s}{r}\right)^{j-k} a_{j}-a_{k}\right)\right|}{\rho}\right)\right]^{p_{k}}=0\right\},
\end{aligned}
$$


where

$$
\begin{aligned}
& \Delta\left(\sum_{\substack{j=k \\
a_{k}+a_{k+1}}}^{n}\left(-\frac{s}{r}\right)^{j-k} a_{j}-a_{k}\right)=\sum_{j=k}^{n} \frac{1}{r}\left(-\frac{s}{r}\right)^{j-k} a_{j}-\sum_{j+1=k}^{n} \frac{1}{r}\left(-\frac{s}{r}\right)^{j+1-k} a_{j+1}- \\
& d_{4}(r, s)=\left\{a=\left(a_{k}\right) \in w: \lim _{n} \sum_{k=0}^{n}\left[u_{k} M_{k}\left(\frac{\left|\left[\frac{1-\left(-\frac{s}{r}\right)^{k+1}}{1+\frac{s}{r}} a_{k}\right]\right|}{\rho}\right)\right]^{p_{k}} \text { exists }\right\} .
\end{aligned}
$$

for all $j, k \in \mathbf{N}$. Then, $D=[b r f, \mathcal{M}, u, p]^{\beta}=\bigcap_{i=1}^{4} d_{i}(r, s)$.

Proof. Let us define the matrix $V=\left(v_{n k}\right)$ via the sequence $z=\left(z_{k}\right) \in w$ by

$$
v_{n k}= \begin{cases}\sum_{k=0}^{n}\left[u_{k} M_{k}\left(\frac{\left|\sum_{j=k}^{n} \frac{1}{r}\left(-\frac{s}{r}\right)^{j-k} z_{j}\right|}{\rho}\right)\right]^{p_{k}}, \quad 0 \leq k \leq n, \\ 0, & k>n,\end{cases}
$$

for all $n, k \in \mathbf{N}$. By considering the relation

$x_{k}=\sum_{k=0}^{n}\left[u_{k} M_{k}\left(\frac{\left|\sum_{j=k}^{n} \frac{1}{r}\left(-\frac{s}{r}\right)^{j-k} y_{j}\right|}{\rho}\right)\right]^{p_{k}}$, we have

$$
\sum_{k=0}^{n} z_{k} x_{k}=\sum_{k=0}^{n}\left[u_{k} M_{k}\left(\frac{\left|\sum_{j=k}^{n} \frac{1}{r}\left(-\frac{s}{r}\right)^{j-k} z_{j} y_{k}\right|}{\rho}\right)\right]^{p_{k}}=(V y)_{n} \quad(n \in \mathbf{N})
$$

From 3.2, we see that $z x=\left(z_{k} x_{k}\right) \in c s$ whenever $x=\left(x_{k}\right) \in[b r f, \mathcal{M}, u, p]$ if and only if $V y \in c$ whenever $y=\left(y_{k}\right) \in b r f$. Then, we have $[b r f, \mathcal{M}, u, p]^{\beta}=$ $\bigcap_{i=1}^{4} d_{i}(r, s)$.

\section{Matrix Transformations}

Başar [1], Kuttner [5] and Lorentz and Zeller [8] have been used the methods of dual summability. Now, let us review these methods.

Let us suppose that the sequences $x=\left(x_{k}\right)$ and $y=\left(y_{k}\right)$ are connected 
with 1.4 and let $A$-transform of the sequence $x=\left(x_{k}\right)$ be $z=\left(z_{k}\right)$ and $B$-transform of the sequence $y=\left(y_{k}\right)$ be $t=\left(t_{k}\right)$, that is,

$$
\begin{aligned}
z_{k} & =(A x)_{k}=\sum_{k} a_{n k} x_{k}, \quad(k \in \mathbf{N}) \\
t_{k} & =(B y)_{k}=\sum_{k} b_{n k} y_{k}, \quad(k \in \mathbf{N}) .
\end{aligned}
$$

Method $B$ is applied to the $B(r, s)$-transform of the sequence $x=\left(x_{k}\right)$ while the method $A$ is directly applied to the terms of the sequence $x=$ $\left(x_{k}\right)$. So it is clear that $A$ and $B$ are essentially different [1]. Let us suppose that the matrix product $B B(r, s)$ exists. If $z_{k}$ turns into $t_{k}$ (or vice versa), under the application of the formal summation by parts, then the methods $A$ and $B$ as in 4.1 and 4.2 are called generalized difference dual type matrices. It means that $B B(r, s)$ exists and is equal to $A$. This condition is equivalent to the following equality:

$$
\begin{gathered}
b_{n k}=\sum_{k=0}^{n}\left[u_{k} M_{k}\left(\frac{\left|\frac{1}{r} \sum_{j=k}^{n}\left(-\frac{s}{r}\right)^{j-k} a_{n j}\right|}{\rho}\right)\right]^{p_{k}} \\
\text { or } a_{n k}=\sum_{k=0}^{n}\left[u_{k} M_{k}\left(\frac{\left|s b_{n, k-1}+r b_{n k}\right|}{\rho}\right)\right]^{p_{k}},
\end{gathered}
$$

for all $n, k \in \mathbf{N}$.

Theorem 4.1. Let $\mu$ be any given sequence space and the matrices $A=$ $\left(a_{n k}\right)$ and $B=\left(b_{n k}\right)$ be generalized difference dual type matrices. Then, $A \in([b r f, \mathcal{M}, u, p]: \mu)$ if and only if $B \in(b r f: \mu)$ and $\left(a_{n k}\right)_{k \in \mathbf{N}} \in$ $[b r f, \mathcal{M}, u, p]^{\beta}$ for all $n \in \mathbf{N}$.

Proof. Let $\mu$ be any sequence space and $A=\left(a_{n k}\right)$ and $B=\left(b_{n k}\right)$ be generalized difference dual type matrices, that is, 4.3 holds. Furthermore, the spaces $[b r f, \mathcal{M}, u, p]$ and $b r f$ are isomorphic. Let $A \in([b r f, \mathcal{M}, u, p]: \mu)$ and $y=\left(y_{k}\right) \in b r f$. Then $B B(r, s)$ exists and $\left(a_{n k}\right)_{k \in \mathbf{N}} \in D$, it means that $\left(b_{n k}\right)_{k \in \mathbf{N}} \in l_{1}$ for each $n \in \mathbf{N}$. Hence, we have

$$
\sum_{k} b_{n k} y_{k}=\sum_{k} a_{n k} x_{k}
$$


for all $n \in \mathbf{N}$ which concluded that $B \in(b r f: \mu)$. On the contrary, let $\left(a_{n k}\right)_{k \in \mathbf{N}} \in[b r f, \mathcal{M}, u, p]^{\beta}$ for each $n \in \mathbf{N}$ and $B \in(b r f: \mu)$ and $x=$ $\left(x_{k}\right) \in[b r f, \mathcal{M}, u, p]$. Then it is clear that $A x$ exists. Thus, we attain from the following equality for all $n \in \mathbf{N}$

$$
\sum_{k=0}^{m} a_{n k} x_{k}=\sum_{k=0}^{m}\left[u_{k} M_{k}\left(\frac{\left|\frac{1}{r} \sum_{j=k}^{n}\left(-\frac{s}{r}\right)^{j-k} a_{j}\right|}{\rho}\right)\right]^{p_{k}} y_{k}=\sum_{k=0}^{m} b_{n k} y_{k}
$$

as $m \rightarrow \infty$ that $A x=B y$ and it is easy to show that $A \in([b r f, \mathcal{M}, u, p]: \mu)$. This completes the proof.

Theorem 4.2. Let us assume that the components of the infinite matrices $A=\left(a_{n k}\right)$ and $E=\left(e_{n k}\right)$ are connected with the following relation

$$
e_{n k}=\sum_{k=0}^{n}\left[u_{k} M_{k}\left(\frac{\left|s a_{n-1, k}+r a_{n k}\right|}{\rho}\right)\right]^{p_{k}},
$$

for all $n \in \mathbf{N}$ and $\mu$ be any given sequence space. Then, $A \in(\mu$ : $[b r f, \mathcal{M}, u, p])$ if and only if $E \in(\mu: b r f)$.

Proof. It is easy to prove.

Now, we list the following conditions;

$$
\sup _{n} \sum_{k=0}^{n}\left[u_{k} M_{k}\left(\frac{\left|\sum_{j=k}^{n} \frac{1}{r}\left(-\frac{s}{r}\right)^{j-k} a_{n j}\right|}{\rho}\right)\right]^{p_{k}}<\infty
$$

$$
\lim _{n}\left[u_{k} M_{k}\left(\frac{\left|\sum_{j=k}^{n} \frac{1}{r}\left(-\frac{s}{r}\right)^{j-k} a_{n j}\right|}{\rho}\right)\right]^{p_{k}}=a_{k} \forall k \in \mathbf{N}
$$

$$
\lim _{n} \sum_{k=0}^{n}\left[u_{k} M_{k}\left(\frac{\left|\Delta\left(\sum_{j=k}^{n} \frac{1}{r}\left(-\frac{s}{r}\right)^{j-k} a_{n j}-a_{k}\right)\right|}{\rho}\right)\right]^{p_{k}}=0
$$


for each fixed $k \in \mathbf{N}$,

where $\Delta\left(\sum_{j=k}^{n} \frac{1}{r}\left(-\frac{s}{r}\right)^{j-k} a_{n j}-a_{k}\right)$

$$
=\sum_{j=k}^{n} \frac{1}{r}\left(-\frac{s}{r}\right)^{j-k} a_{n j}-\sum_{j+1=k}^{n} \frac{1}{r}\left(-\frac{s}{r}\right)^{j+1-k} a_{n, j+1}-a_{k}+a_{k+1} \text {. }
$$

$$
\operatorname{brf}-\lim _{n}\left[u_{k} M_{k}\left(\frac{\left|\sum_{j=k}^{n} \frac{1}{r}\left(-\frac{s}{r}\right)^{j-k} a_{n j}\right|}{\rho}\right)\right]^{p_{k}}=a_{k}
$$

exists for each fixed $k \in \mathbf{N}$,

$$
\sup _{n} \sum_{k}\left[u_{k} M_{k}\left(\frac{\left|s a_{n-1, k}+r a_{n k}\right|}{\rho}\right)\right]^{p_{k}}<\infty
$$

$$
\operatorname{brf}-\lim _{n}\left[u_{k} M_{k}\left(\frac{\left|s a_{n-1, k}+r a_{n k}\right|}{\rho}\right)\right]^{p_{k}}=a_{k},
$$

exists for each $k \in \mathbf{N}$,

$$
b r f-\lim _{n}\left[u_{k} M_{k}\left(\frac{\left|s a_{n-1, k}+r a_{n k}\right|}{\rho}\right)\right]^{p_{k}}=a,
$$

By using the lemmas of [21] and Theorems 4.1 and 4.2, we derive the following results:

Corollary 4.3. The following statements hold:

(i) $A=\left(a_{n k}\right) \in\left([b r f, \mathcal{M}, u, p]: l_{\infty}\right)$ if and only if $\left(a_{n k}\right)_{k \in \mathbf{N}} \in[b r f, \mathcal{M}, u, p]^{\beta}$ for all $n \in \mathbf{N}$ and 4.6 holds.

(ii) $A=\left(a_{n k}\right) \in([b r f, \mathcal{M}, u, p]: c)$ if and only if $\left(a_{n k}\right)_{k \in \mathbf{N}} \in[b r f, \mathcal{M}, u, p]^{\beta}$ for all $n \in \mathbf{N}$ and 4.6, 4.7, 4.8 and 4.9 hold.

(iii) $A=\left(a_{n k}\right) \in\left(l_{\infty}:[b r f, \mathcal{M}, u, p]\right)$ if and only if 4.10, 4.11 and 4.12 hold. (iv) $A=\left(a_{n k}\right) \in(c:[b r f, \mathcal{M}, u, p])$ if and only if 4.11 and 4.12 hold. 


\section{Acknowledgement:}

The authors are thankful to the reviewer(s) for their valuable comments and suggestions which improved the presentation of the paper. The corresponding author thanks the Council of Scientific and Industrial Research (CSIR), India for partial support under Grant No. 25(0288)/18/EMR-II, dated 24/05/2018.

\section{References}

[1] F. Bașar, "Matrix transformations between certain sequence spaces of Xp and lp", Soochow journal of mathematics, vol. 26, no. 2, pp. 191-204, 2000.

[2] F. Bașar, Summability theory and its applications. Istanbul: Bentham ebooks, 2012, doi: 10.2174/97816080545231120101.

[3] F. Başar and M. Kirişci, "Almost convergence and generalized difference matrix", Computers \& mathematics with applications, vol. 61, no. 3, pp. 602-611, Feb. 2011, doi: 10.1016/j.camwa.2010.12.006.

[4] H. Dutta and F. Başar, "A generalization of Orlicz sequence spaces by Cesàro mean of order one", Acta mathematica universitatis comenianae, vol. 80, no. 2, pp. 185-200, (2011). [On line]. Available: http://bitly/2nbb9uk

[5] B. Kuttner, "On dual summability methods", Mathematical proceedings of the cambridge philosophical society, vol. 71, no. 1, pp. 67-73, Jan. 1972, doi: $10.1017 /$ S0305004100050283.

[6] J. Lindenstrauss and L. Tzafriri, "On Orlicz sequence spaces", Israel journal of mathematics, vol. 10, no. 3, pp. 379-390, Sep. 1971, doi: 10.1007/BF02771656.

[7] G. Lorentz, "A contribution to the theory of divergent sequences", Acta mathematica, vol. 80, pp. 167-190,1948, doi: 10.1007/BF02393648.

[8] G. Lorentz and K. Zeller, "Summation of sequences and summation of series", Proceedings of the american mathematical society, vol. 15, no. 5, pp. 743-746, Oct. 1964, doi: 10.2307/2034590.

[9] L. Maligranda, Orlicz spaces and interpolation, vol. 5. Campinas, SP: Universidade Estadual de Campinas, 1989.

[10] M. Mursaleen, S. K. Sharma, S. A. Mohiuddine, and A. Kllıçman, "New difference sequence spaces defined by Musielak-Orlicz function", Abstract and applied analysis, vol. 2014, ID 691632, Jul. 2014, doi:10.1155/2014/691632.

[11] M. Mursaleen, "Generalized spaces of difference sequences", Journal of mathematical analysis and applications, vol. 203, no. 3, pp. 738-745, Nov. 1996, doi: 10.1006/jmaa.1996.0409.

[12] J. Musielak, Orlicz spaces and modular spaces, vol. 1034. Berlin: Springer, 1983, doi: $10.1007 / \mathrm{BFb} 0072210$.

[13] G. Petersen, Regular matrix transformations. London: McGrawHill, 1966. 
[14] K. Raj, A. Choudhary, and C. Sharma, "Almost strongly Orlicz double sequence spaces of regular matrices and their applications to statistical convergence", Asian-European journal of mathematics, vol. 11, no. 05, ID 1850073, 2018, doi: 10.1142/S1793557118500730.

[15] K. Raj and A. Kulıçman, "On certain generalized paranormed spaces", Journal of inequalities and applications, vol. 2015, no. 1, Jan. 2015, doi: 10.1186/s13660-015-0565-z.

[16] K. Raj and C. Sharma, "Applications of strongly convergent sequences to Fourier series by means of modulus functions , Acta mathematica hungarica, vol. 150, no. 2, pp. 396-411, Aug. 2016, doi: 10.1007/s10474-0160655-5.

[17] K. Raj and S. Sharma, "Some multiplier generalized difference sequence spaces over n-normed spaces defined by a Musielak-Orlicz function", Siberian advances in mathematics, vol. 24, no. 3, pp. 193-203, Aug. 2014, doi: 10.3103/S1055134414030067.

[18] A. Sönmez, "Almost convergence and triple band matrix", Mathematical and computer modelling, vol. 57, no. 9-10, pp. 2393-2402, May 2013, doi: 10.1016/j.mcm.2011.11.079

[19] A. Wilansky, Ed., Summability through functional analysis, vol. 85. Amsterdam: Elsevier, 1984. [On line]. Available: https://bit.ly/2npkCHC

[20] M. Yeşilkayagil and F. Başar, "Spaces of $A \lambda$-almost null and $A \lambda$-almost convergent sequences", Journal of the egyptian mathematical society, vol. 23, no. 1, pp. 119-126, Apr. 2015, doi: 10.1016/j.joems.2014.01.013.

[21] Z. Zararsiz and M. Sengönül, On the almost convergence, Doctoral Thesis, Nevșehir Haci Bektaş Veli University, 2019.

[22] Z. Zararsiz, "On the extensions of the almost convergence idea and core theorems", Journal of nonlinear sciences and applications, vol. 09, no. 01, pp. 112-125, Jan. 2016, doi: 10.22436/jnsa.009.01.11. 